\begin{tabular}{|c|}
\hline 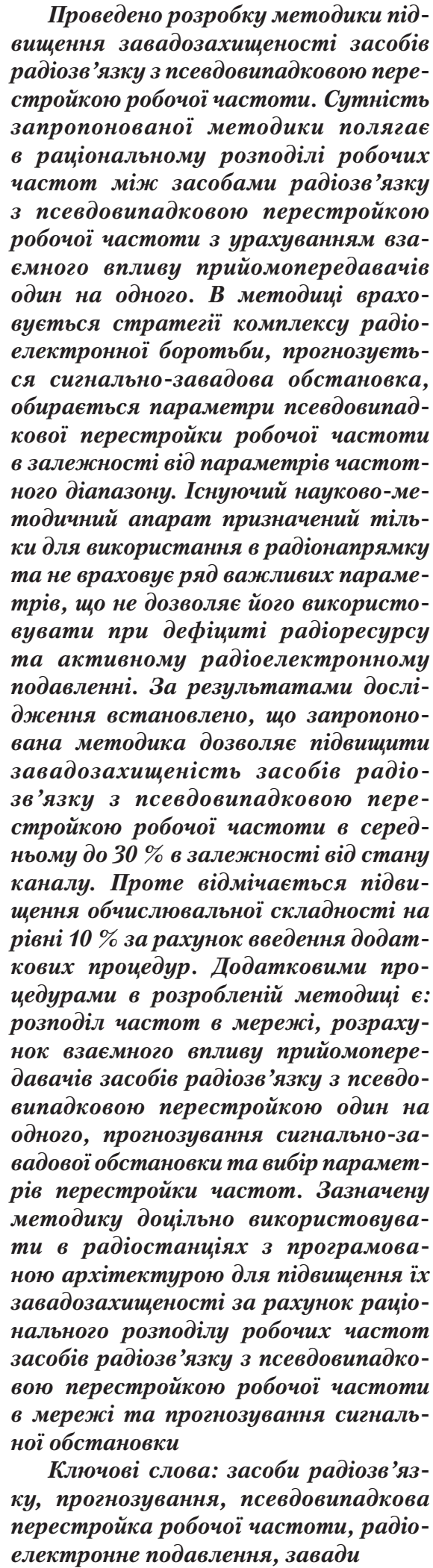 \\
\hline
\end{tabular}

口-

\section{DEVELOPMENT OF A METHOD OF INCREASING THE INTERFERENCE IMMUNITY OF FREQUENCY- HOPPING SPREAD SPECTRUM RADIO COMMUNICATION DEVICES}

O. Kuvshy nov

Doctor of Technical Sciences, Professor, Deputy Chief Educational-Scientific Institute*

A. Shyshat sk y i

$\mathrm{PhD}$, Chief of Research Laboratory Research Laboratory of Electronic Warfare Development*** E-mail: ierikon12@gmail.com

O. Z h u k

$\mathrm{PhD}$, Associate Professor, Leading Researcher Scientific Department****

R. B i eliakov

$\mathrm{PhD}$

Department of Technical and Metrological Support****

Ye.Prokopenko $\mathrm{PhD}$

Institute of Information Technology*

O. Le on t i e v

Doctor of Technical Sciences, Professor, Chief Researcher Scientific Center of Air Forces of Ukraine Ivan Kozhedub Kharkiv National Air Force University Sumska str., $77 / 79$, Kharkiv, Ukraine, 61023

R. Z h y vot ov sk y i

$\mathrm{PhD}$, Chief of Research Department**

H. Drobakha

Doctor of Military Sciences, Professor, Chief Researcher Research Center Service and Combat Activity National Academy of the National Guard of Ukraine Zakhysnykiv Ukrainy sq., 3, Kharkiv, Ukraine, 61001

I. Rom a n e n ko Doctor of Technical Sciences, Professor, Leading Researcher Scientific-Research Department***

S. P e t r u k

Deputy Chief of Research Department** *Ivan Chernyakhovsky National Defense University of Ukraine

Povitroflotskyi ave., 28, Kyiv, Ukraine, 03049

** Research Department of Development of Anti-Aircraft Missile Systems and Complexes*** ***Central Scientifically-Research Institute of Arming and Military Equipment of the Armed Forces of Ukraine Povitrofloski ave., 28, Kyiv, Ukraine, 03049 $\star * * *$ Military Institute of Telecommunication and Information Technologies named after Heroes Krut Moskovska str., 45/1, Kyiv, Ukraine, 01011

interference, as well as provision of multi-station access when working in packet radio networks, can best be solved by using spread spectrum signals in radio communication devices (RCD) [1].
The problem of providing reliable communication in the conditions of the influence of organized and unintentional 
The essence of the spread spectrum method consists in the following:

- spread spectrum is a transmission method in which the signal occupies a bandwidth which is wider than the band that is minimally necessary for the transmission of information;

- signal bandwidth spreading is provided by a special code that does not depend on the transmitted information;

- for the next compression of the signal bandwidth and data recovery in the receiver, the RCD also uses a special code, similar to the code in the RCD transmitter and synchronized with it.

One type of spectrum expansion used in modern RCD is the frequency-hopping spread spectrum (FHSS) method [1-4].

For the FHSS method, the principle of combating noise is to place an information signal with a small dimension in a high-dimensional signal space. In such conditions, the noise generator must distribute the limited power of the interference over the entire frequency range, thereby creating a small spectral density of interference power. Otherwise, the noise generator should use the entire power of the transmitter in a limited frequency range, leaving part of the RCD bandwidth range free of interference.

The typical shortcomings of the FHSS method are the low speed of data transmission and the absence of mechanisms to combat fading, resulting in error packets in the communication channel.

Nowadays, active scientific research is being carried out on increasing the interference immunity of FHSS RCD, increasing the use of radio resources and electromagnetic compatibility. This is due to an increase in the technological level of the devices of electronic warfare and the lack of radio resources of special users.

\section{Literature review and problem statement}

While designing adaptive radio communication systems, depending on the destination, the task consists in optimizing one of the performance indicators under the established restrictions to others.

The article [1] is devoted to the disclosure of the properties of the FHSS method. It discusses in detail the advantages and disadvantages of this method, the basic mathematical models and analyzes the interference immunity of this method. However, no specific mechanisms for increasing the interference immunity of this method are proposed. The whole research is based on the use of frequency shift keying signals in FHSS, without considering other types of signal-code designs.

In the article [2], an adaptive algorithm for the selection of operating frequencies for FHSS RCD in the conditions of intentional and unintended interference is considered. This algorithm is implemented on the basis of the theory-game approach. The disadvantage of this algorithm is that it is designed to work in the radio direction and it is not suitable for radio network operation.

In the article [3], the method of automatic determination of the time parameters of FHSS radio signals on the background of narrowband noise is presented. The method is based on the analysis of the two-dimensional energy distribution of the signal mixture obtained using the window-modified Welch periodogram. The method allows detecting and removing outside narrowband radiation from the calculations, and applying an algorithm for detecting abnormal values, filtering the false-defined duration of the frequency elements. The indicated method is not suitable for the work with other types of interference and in the FHSS RCD radio network.

In the article [4] a method of dynamic selection of workers for industrial FHSS RCD is proposed. The essence of the proposed method is to evaluate the available frequency range by several standard indicators (probability of bit error and sig$\mathrm{nal} /$ noise ratio) and the selection of the best frequency subchannels for operation. The method is not intended to work in the conditions of intentional interference and does not allow for the mutual influence of transceivers on each other.

In the article [5] a technique for the formation of FHSS signals in the conditions of the effect of intentional noise disturbances is proposed. The essence of the technique is to select the desired bandwidth of the FHSS signal (hopset), which provides the desired quality of information transmission. The author of this work limited himself to considering only one type of deliberate disturbance and does not take into account the mutual influence of the RCD transmitters on each other.

In the article [6], the development of a signal formation method in FHSS radio devices in the transmission of speech in the conditions of interference influence in response is carried out. The essence of the methodology is the location of the characters of speech frames in the interval of the frequency elements of the signal with the RCD, in which the interference in response affects the minimally important characters for speech reproduction, regardless of the type of the transmitted signals.

In the article [7], an advanced method for selecting rational values of the parameters of multi-antenna systems of military frequency-hopping spread spectrum radio communication, depending on the signal-interference situation, was proposed. This method allows predicting the signal-interference situation, choosing the most suitable working frequencies. This technique also allows one not only to change the speed of the adjustment of the operating frequency but also to change the appearance and duration of the expansion sequence, as well as the initial filling of the forming polynomial. The disadvantage of this methodology is that it does not allow determining the mutual influence of the transceivers and it is suitable only for the radio direction.

In the article [8], an algorithm for determining the time delay of FHSS signals was developed. The essence of the algorithm is to split the FHSS signal into separate frequency channels that correspond to the corresponding carrier frequencies. After that, the calculation of the mutual function of uncertainty in each channel occurs. The specified algorithm allows increasing the interference immunity of FHSS RCD, but it can not be used for the work in the radio network.

In the article [9], a method for finding FHSS signals and estimating the carrier frequency is proposed. The indicated method allows increasing the interference immunity by more accurate estimation of carrier parameters and finding FHSS frequency elements. However, this method does not allow predicting the state of the frequency range.

In the article [10], a method for assessing the state of the FHSS channel, designed to detect signals against the background of interference is developed. In the indicated method, there is no prediction of the state of the frequency range.

In the article [11], the simulation of the tactical systems of broadband communication was carried out. In the given work, the influence of various types of intentional interference on its interference immunity is determined. It is proposed to deal with different types of signal modulation and correcting 
codes to control interference. However, other mechanisms for increasing interference immunity are not proposed.

In the article [12], the development of a method for finding FHSS signals is carried out. The indicated method allows finding FHSS signals but does not allow the distribution of frequencies in the network and the prediction of the frequency range state.

However, the scientific results presented in the known publications [1-12] have a number of shortcomings, namely:

- the choice of operating frequencies for FHSS RCD occurs without taking into account the strategy of electronic countermeasure (ECM) complexes and without taking into account the influence of the RCD transmitters on each other;

- the frequency distribution between FHSS radio communication devices is carried out in the radio direction without taking into account the frequencies used in the radio communication system;

- planning of the use of frequencies between the RCDs in the system takes place without taking into account the results of prediction of the effect of intentional interference on separate sections of the frequency range;

- control of the FHSS parameters is carried out only by changing the speed of the adjustment of the operating frequency (without changing the law of the adjustment of the operating frequency);

- the impact of only one type of noise is taken into account;

- a limited list of signal-code designs is used.

From the above analysis, there is a contradiction between science and practice, which is as follows:

- in science, existing methods for increasing the interference immunity of FHSS radio communication devices do not take into account the influence of the RCD transmitters on each other, are not able to predict the load of the radio frequency spectrum and are not able to change the law of the adjustment of the operating frequency during the radio communication session, which significantly reduces the interference immunity of the RCD;

- in practice, a large number of destabilizing factors must be taken into account in order to increase the interference immunity of the RCD.

\section{The aim and objectives of the study}

The aim of the study is to develop a methodology for increasing the interference immunity of FHSS radio communication devices. To achieve this aim, the following objectives were accomplished:

- to evaluate the possibilities of the system of electronic countermeasure of FHSS radio communication devices;

- to develop the algorithm of the method of increasing the interference immunity of FHSS radio communication devices;

- to determine the criteria for assessing the effectiveness of the proposed methodology and assess its effectiveness.

\section{1. Estimation of parameters of the electronic coun-} termeasure system

It should be mentioned that for the RCDs, one of the most unfavorable obstacles is the retransmitted interference (interference in response) [1, 6, 7]. The range of ECM devices depends on many factors, including the power of radio transmitting devices of radio communication and $\mathrm{ECM}$ equipment. In addition, the range of ECM devices is influenced by the intensity of interference from local objects, the terrestrial (water) surface and non-earthly sources, the nature of the radiation and scattering of electromagnetic waves by the targets observed by RCD. Considering all these factors is extremely difficult. In this regard, the suppression range of RCD and the required power of the ECM devices are evaluated mathematically by averaged parameters and specified in the simulation process.

We shall evaluate the time possibilities of the interference station with the provision of retransmitted interference to suppress the radio communication system (RCS).

The termination of the retransmitted interference must coincide with the time at which the radiation of radio equipment signals ceases.

The time required to perform the listed functions (the response time of the retransmitted interference station) $\Delta t_{r}$ must be small enough so that the retransmitted interference has time to affect the radio receiver until the RCS transmitter is rebuilt to a different frequency.

The result of the effect of retransmitted interferences on the receiver of the RCD in the general case can be estimated by the magnitude of the average probability of error per bit of information $P_{b}$ having the form [7]:

$$
P_{e r}=\rho P_{1}\left(\frac{E_{s}}{G_{\Sigma}}\right)+(1-\rho) P_{2}\left(\frac{E_{s}}{G_{\Sigma}}\right),
$$

where $P_{1}$ is the average probability of a bit error in the effect of retransmitted interference; $P_{2}$ is the average probability of a bit error in the absence of retransmitted interference at the input of the demodulator, where $G_{\mathrm{S}}$ is the total spectral density of white Gaussian noise and intentional noise, $r$ is the coefficient characterizing the part of the frequency element affected by the interference (overlap factor), $0 \leq \rho \leq 1$.

In promising stations of retransmitted interference in the USW range using the latest technological advances and high-speed microprocessor technology in the ECM equipment, the minimum response time $\Delta t_{r \min }$ can be $100 \mathrm{~ms}$ or less $[6,7]$. In these conditions, an important parameter of the radio devices (in terms of interference immunity) is the actual operating time at the same frequency $\Delta t_{o p}$.

The overlapping factor of the signal by transmitted interference depends not only on the response time $\Delta t_{r}$, but also on the relative location (topology) of the RCS transmitter (TRS) and the receiver (RCV), as well as the station of retransmitted interference on the location, which determines the delay time of interference $\Delta t_{d}$ (Fig. 1).

If the radio device's operating time at the same frequency $\Delta t_{o p}$ is less than the total response time $\Delta t_{r}$ of the station of interference and the delay time of interference $\Delta t_{d}$, then the retransmitted interference is ineffective. In this case, the permissible operating time $\Delta t_{o p}$ of the receiver without the influence of noise is equal to.

$$
\Delta t_{o p} \leq \Delta t_{r}+\Delta t_{d} ; \quad \Delta t_{d}=\frac{1}{C_{r}}\left(r_{1}+r_{2}-r_{12}\right),
$$

where $r_{1}$ is the distance from the station of retransmitted interference to the RCS transmitter, $r_{2}$ is the distance from the station of retransmitted interference to the RCS receiver, $r_{12}$ is the distance between the RCS transmitter and receiver, $C_{r}$ is the speed of radio wave propagation.

The closer the SRC receiver is to the station of retransmitted interference, the less the allowable time for its operation $\Delta t_{o p}$ without interference. 


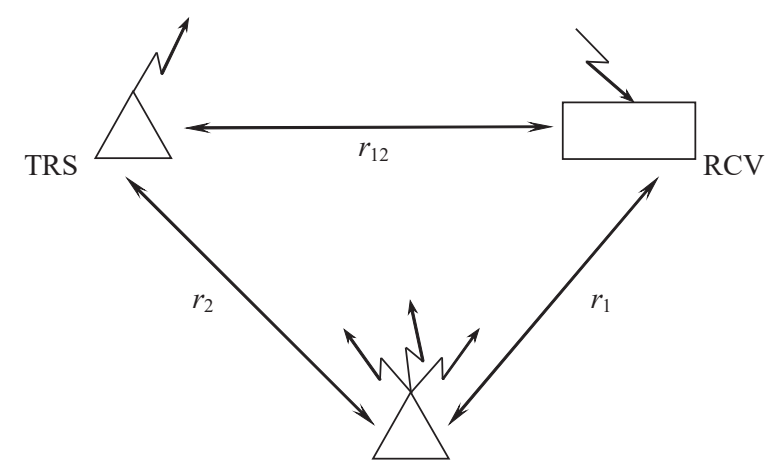

station of the retransmitted interferences

Fig. 1. Relevant location of the station of retransmitted interference and RCS elements

The effective impact of retransmitted interference at its sufficient power on the RCS receiver is achieved with the ratio:

$$
(1-\rho) \Delta t_{o p} \geq \frac{r_{1}+r_{2}-r_{12}}{C_{r}}+\Delta t_{r}
$$

Using expression (3), it is possible to estimate the time capabilities of the retransmitted interference station in the RCS suppression with different duration of work at one frequency.

In the article [6], the dependencies of the permissible operating time $\Delta t_{o p}$ of the RCS receiver without the retransmitted disturbances influence on $r=r_{1}+r_{2}-r_{12}$ distance for different values of the overlap factor $r$ are given. With the right choice of the frequency adjustment rate of $\operatorname{RCS} v_{a d j}=1 / \Delta t_{o p}$, it is possible to completely get rid of retransmitted interference.

Therefore, it is necessary to improve the existing scientific and methodical apparatus for controlling the parameters of FHSS radio communication systems and devices.

4.2. Essence of the methodology and the algorithm of its implementation

The essence of the methodology is an adaptive selection of signal parameters of FHSS radio communication systems, which are optimal for the maximum criterion of the energy efficiency indicator with restrictions on the probability of erroneous reception, depending on the interference situation in the communication channel.

Setting objectives.

Given: parameters of the transmitter and communication channel $\Psi=\left\{\psi_{i}\right\}, i=\overline{1,9}$, where $\psi_{1} \ldots \psi_{9}$ is the rate of the FHSS $v_{h o p}$, the maximum power of the useful signal $P_{s \max }$, the process gain $K_{s}$, the rate of information transmission $v_{i}$, the bandwidth of the channel $\Delta F$, a set of correction codes with corresponding parameters.

It is necessary: to determine the values of the signal parameters (the rate of the FHSS, the parameters of the SCC, the process gain, the transmitter power), at which the energy efficiency $\beta_{E}$ is maximized, while fulfilling the limits on the probability of erroneous reception in the channel $P_{e r} \geq P_{\text {er prob }}$.

Limitation: the speed of correction code $R=0.5-0.9$; the dimension of the signal ensemble $2 \leq M \leq 16$; the process gain $128 \leq K_{\mathrm{s}} \leq 1,024$; the maximum acceptable probability of error signal reception $P_{\text {er prob }}=10^{-5}$, the rate of the FHSS $100 \leq v_{h o p} \leq 10,000$. Type of interference frequency shift keying partial band noise interference, frequency shift keying noise interference, retransmitted interference, which is an actual copy of a useful signal with the time displacement.

The tasks of determining the parameters of the FHSS signal with the maximum energy efficiency indicators are reduced to a typical optimization problem. The system of equations for solving the optimization problem has the form:

$$
\left\{\begin{array}{l}
\beta_{E}=F_{1}\left(v_{i}, \Delta F, M, v_{A}, R, d, P_{s}, K_{s}\right) \rightarrow \max \\
P_{e r}=F_{2}\left(P_{s}, M, R, d, K_{s}, \gamma, \rho\right) \leq P_{\text {er prob }}
\end{array}\right.
$$

where $P_{S}$ is the signal power, $M$ is the dimension of the signal ensemble, $R$ is the speed of the correction code $(R=k / n), k$ is the number of information bits in the code combination of length $n$, $d$ is the code distance value, $\Delta F$ is the bandwidth of the channel.

The system of equations for solving the optimization problem can be transformed into the form:

$$
\left\{\begin{array}{l}
\beta=\frac{v_{V}}{P_{s} / G_{n}}=\max ; 100 \leq v_{c h} \leq 10000 ; 128 \leq K_{s} \leq 1024 ; \\
P_{e r}=\sum_{j=s_{c o r}+1}^{n} C_{n}^{j} P_{e r}^{j}\left(1-P_{e r}\right)^{n-j} \leq P_{e r ~ p r o b} ; 2 \leq M \leq 16,
\end{array}\right.
$$

where $s_{\text {cor }}=(d-1) / 2$ is the multiplicity of errors that the code corrects, $j$ is the multiplicity of the error in the block of $n$ elements, $C_{n}^{j}=n ! / j !(n-j) !$ is the binomial coefficient, which is equal to the number of different combinations $j$ of errors in the block of $n$ symbols.

The method of selecting the parameter values of signals of FHSS RCD, the algorithm of which is presented in Fig. 2, consists of the following steps.

1. Entering the output data. The parameters of the transmitter and the communication channel $\Psi=\left\{\psi_{i}\right\}, i=\overline{1, m}$, as well as the given speed of information transmission $v_{\text {per }}$ are entered.

2. Distribution of frequency resource among radio communication devices.

Recently, the urgent task of ensuring the quality of radio equipment functioning in common and adjacent bands of radio frequencies appears, which requires the development of new approaches to the analysis of electromagnetic compatibility of radio equipment [15]. General approaches and separate methods of solving problems of this class are set out in the recommendations of the International telecommunication union (ITU) [15]. But the proposed recommendations do not provide a comprehensive solution to the problem of electromagnetic compatibility (EMC) analysis of special purpose radio communication devices.

Moving radio service involves the use of radio electronic devices (RED), the location of which in the area is changing, which complicates the EMC analysis [17].

The proposed procedure involves the use of a calculation model containing a pair and group estimation with a simple logic of the mutual effect of RED in a group that is based on a probabilistic (statistical) approach to evaluation [18]. Simple logic suggests that each of the RCD in a group can be considered as functionally independent from the other devices. The calculation of EMC of RED is carried out in the following order:

1. Selection of RES on a territorial basis, located within a limited research area;

2. Selection of RES, selected on a territorial basis, which potentially can interfere with the new frequency assignment of the REF on a frequency basis:

2. 1. Determination of possible interference sources over the main channel; 
2. 2. Determination of possible interference sources on the first adjacent channel;

2.3. Determination of possible sources of interference intermodulation of the 3rd order;

2.4. Calculation of the interference level at the receiver input:

- on the main channel;

- on the first adjacent channel;

- intermodulation of the 3rd order.

3. Analysis of the results obtained and decision making based on the results of calculations on the possibility of frequency assignment, taking into account the effects of multiple noises or the need to choose another frequency.

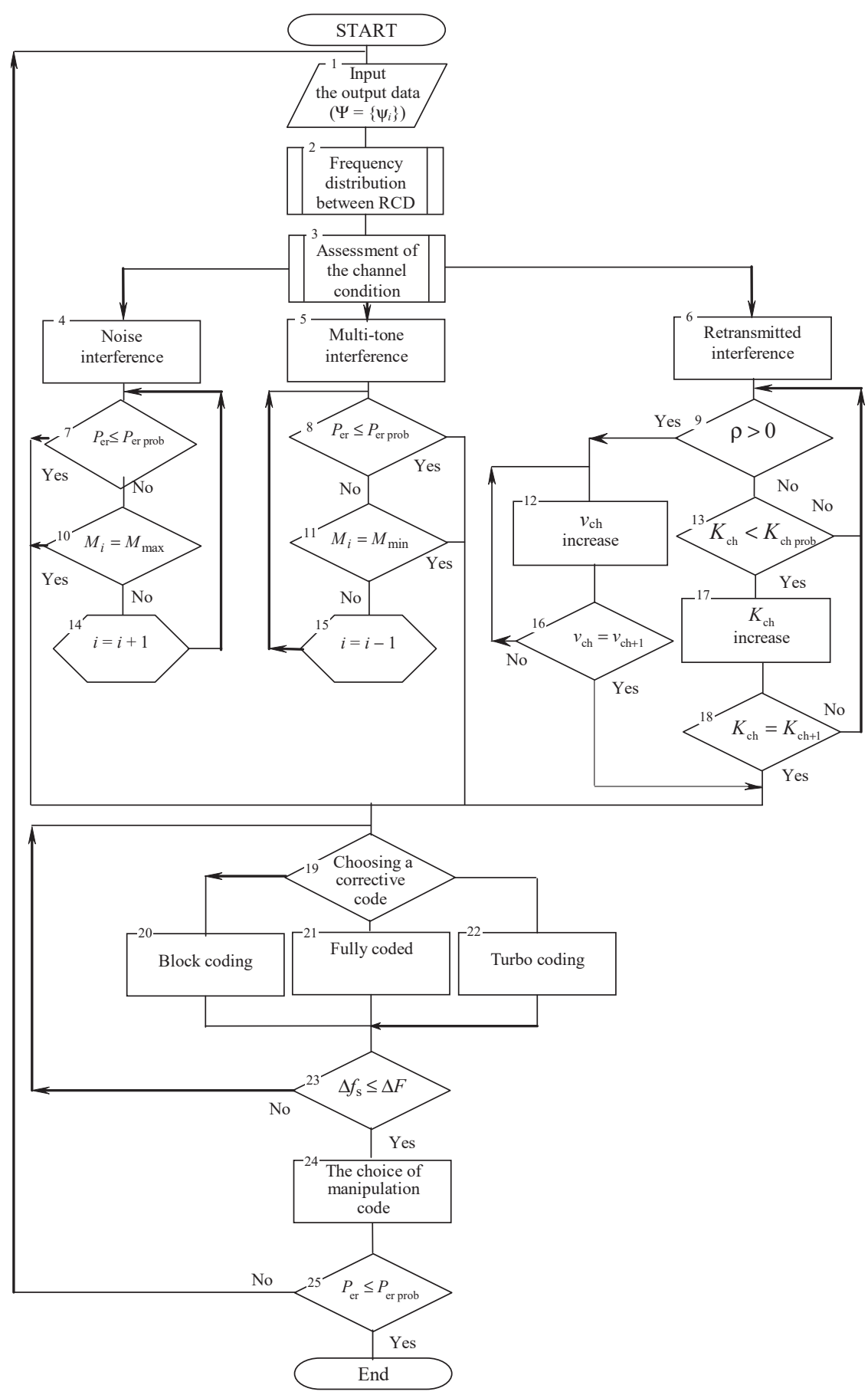

Fig. 2. Scheme of the algorithm for the method of controlling the parameters of RCS signals under the influence of intentional interference
To optimize the calculations, the analysis of EMC is proposed in two stages: preliminary and detailed. In the preliminary step, pre-selection and analysis of EMC for the specified frequencies are carried out.

In the detailed analysis, the EMC calculation is carried out taking into account the influence of the side radiation, the possibilities of blocking the receiver and the influence of intermodulation interference products.

The general algorithm for the assignment of operating frequencies and the EMC analysis for the RCD (Fig. 3) contains:

1. Entering the initial data.

2. Choosing a free frequency $f_{\text {new }}$ within the calculation zone.

3. Selection of existing RED within the calculation zone. 4. Selection of noisy RED according to the frequency criterion.

5. Calculation of the interference level $P_{i}$ at the receiver input in the main and adjacent channels.

6. Analysis of the preliminary calculation results.

7. Calculation of the level of interference from the transmitter.

8. Analysis of the results obtained in the calculation.

9. Selection of RED according to the criterion of proximity is carried out. RED for further calculations are selected within the radius of the service area.

10 . The calculation of possible interference from the stations selected in the previous stage by adjacent and mirror channels, harmonic interference and intermodulation interference is carried out. The calculation of total interference $P_{i \Sigma}$ for each station within the defined zone is carried out in accordance with the procedure outlined in [18] and contains the following steps:

- calculation (measurement) of values and forms of interference spectra at the receiver input;

- calculation of the total power of interference at the input of the radio frequency amplifier;

- definition of interference connections formed by intermodulation products at the output of the radio frequency amplifier at the frequencies of the main and mirror channels;

- determination of values and forms of spectra of intermodulation products at the output of the radio frequency amplifier;

- definition of the total power of intermodulation products at the output of the intermediate frequency filter;

- comparison of the calculated values of the total interference power with allowable.

11. If as a result of new frequency assignment, total interference exceeds the threshold, transition to the selection of a new frequency is carried out (stage 2). 
12. A separate analysis of the object electromagnetic compatibility for the planned RCD receiver is carried out, similarly to stage 10 .

13. The required protective relation is determined for the planned RCD receiver, based on the frequency selectivity of the receiver.

14. If the total interference exceeds a certain threshold, transition to the selection of a new frequency is carried out (stage 2).

15. A positive conclusion about the possibility of frequency assignment is made.

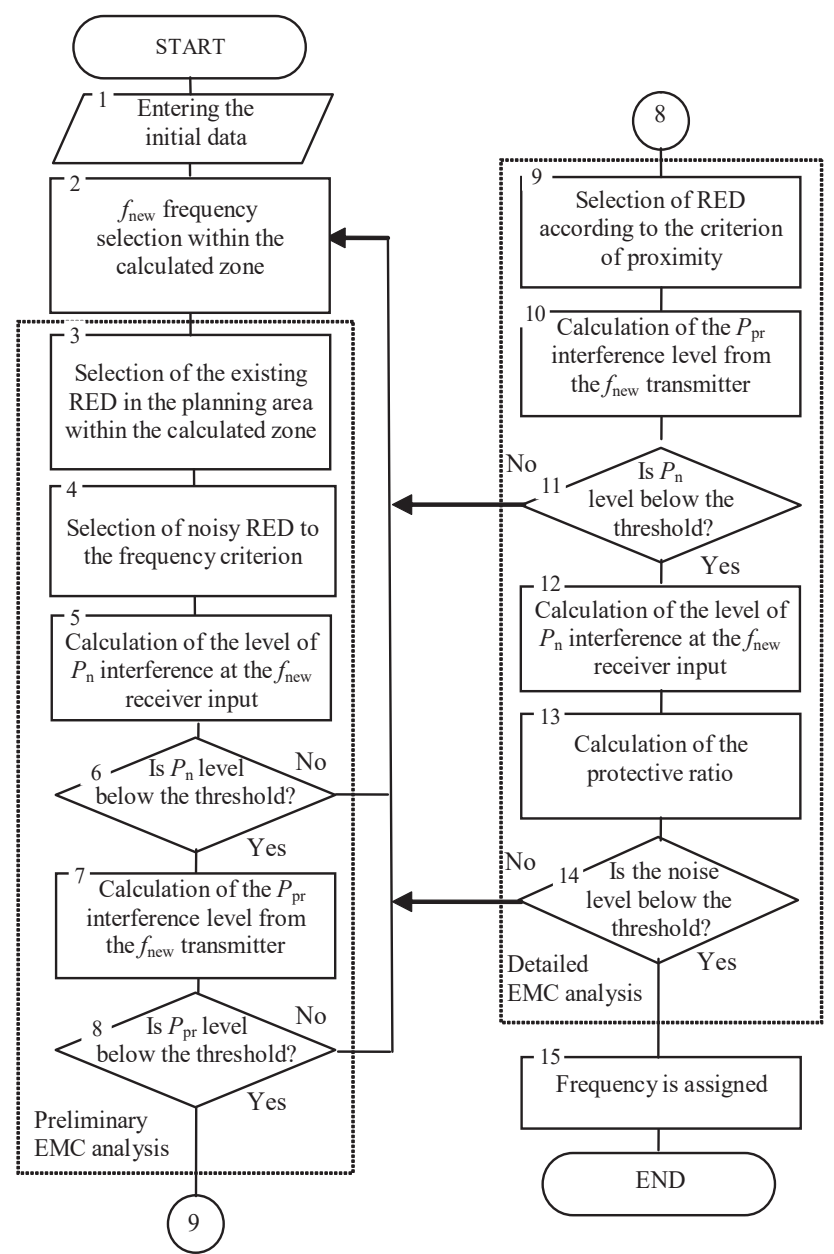

Fig. 3. Algorithm of the procedure for selecting the operating frequencies and analysis of electromagnetic compatibility

Forecasting of signal-interference situation. The procedure described differs from those known because it additionally comprises operations:

- recirculation of input data for one count;

- resampling of the output process on the logarithmic time scale;

- finding the energy spectrum of the received signal, determining the response;

- finding the entropy of the energy spectrum of the corresponding sample, which is subject to resampling;

- calculating the maximum value of entropy response;

- finding the forecast for implementing the maximum entropy value;

- resampling the result of forecasting on the exponential time scale.
The procedure for forecasting the signal-interference situation in the frequency range has the following sequence of actions [7]:

1. Entering of initial data.

2. Time compression of the predicted process, which is necessary for real-time signal processing. At the same time, the implementation is updated by a single countdown at each step. Thus, a class of implementations, which differ from each other by one count, is formed. For forming a class of discrete counts, each implementation is subjected to the logarithmation and sampling operation.

The maximum entropy value is determined according to the ratio:

$$
H(f)=-\int_{-1 / 2}^{1 / 2} \ln \left(\frac{X(f)}{\int_{-1 / 2}^{1 / 2}(X(f)) \mathrm{d} f}\right) \mathrm{d} f,
$$

where

$$
X_{n}(f)=\frac{X(f)}{\int_{-1 / 2}^{1 / 2}(X(f)) \mathrm{d} f}
$$

is the normalized energy spectrum of the sample,

$$
X(f)=\sum_{n=-\infty}^{\infty} r_{s s}(n) \exp (-2 \pi f n)
$$

$r_{s s}(n)$ is the correlation function of the process.

Using the proposed procedure allows you to get a more accurate forecast than using other known procedures.

The choice of operating frequencies for radio communications in the conditions of deliberate interference is carried out with a minimum probability of bit error. This algorithm is based on the representation of the process of electronic warfare in the form of two antagonistic systems. At the same time, a management decision is made taking into account the analysis of the electronic environment and the criterion for minimizing the probability of bit error. In order to implement this algorithm, the use of the vector of the coefficient of utilization of operating frequencies by each antagonistic system (factor of frequency sub-channel overlapping by interference) was chosen.

The main stages of the algorithm:

1. Determination of the type and characteristics of intentional interference.

2. Checking the value of the overlapping factor.

3. Formation of the game matrix of the radio-electronic conflict.

4. Formalization of the linear programming problem.

5. Checking the optimal control of the choice of operating frequencies.

3. Assessment of the state of the communication channel. At this stage, with the help of known methods [18-23], the spatial parameters of FHSS radio sources are estimated. In addition, the type and characteristics of intentional interference affecting the receiver input (noise, multi-tone interference or interference in response) are determined for a particular radio device.

4. Selection of the signal parameters. Depending on the type of interference acting on the receiver input, the positioning of the signal ensemble, frequency adjustment rate and the magnitude of the process gain are selected. 
In the articles [21-23], under the influence of deliberate interference, signal-code designs were chosen by maximizing the energy efficiency with restrictions on the value of the interference immunity coefficient for the average values of the signal-to-noise ratio in the discrete symmetric channel. They use analytical dependencies for calculating the bandwidth of a discrete symmetric channel, which is are effective only for average signal/interference ratios corresponding to the probability of bit decoding error $P_{e r}<10^{-6}$. These works do not take into account the fact that FHSS radio communication devices in the conditions of intentional interference can operate at the low signal-to-noise ratio in the channel and the probability of bit decoding error of $10^{-1} \ldots 10^{-4}$.

Considering that algorithms for decoding turbo codes are designed for «soft» input, it is necessary to use a «soft» output demodulator in the RCD and consider discrete-continuous communication channels [24].

In order to calculate the probability of bit error $P_{e r}$, it is recommended to use approximate formulas for large signal/noise ratio $Q_{0}^{2}$ in the channel [24]. To analyze the characteristics of noise immunity of the turbo code used in channels with the increased noise level, as well as in the presence of intentional interference in the communication channel, these formulas become inadequate in the calculation.

Therefore, we will use mathematical relationships to calculate the probabilities of bit error for different types of signals and intentional interference used to suppress FHSS RCD given in the article [22].

As it is shown in [25], in the vast majority of cases it is possible to identify several possible scenarios of the interference situation. These scenarios can be matched with $N$ different signal-code designs, which are appropriate to choose based on the efficiency parameters of the RCD under the influence of various types of intentional interference.

Thus, when the radio communication device is exposed to barrage jamming and partial band noise interference with increasing multiplicity of manipulation, the interference immunity of signal reception is improved (blocks 6, 9, 13). Under the influence of multi-tone interference to reduce the probability of bit error, the position of the signal ensemble must be reduced (blocks 7, 10, 14).

In promising stations of retransmitted interference in the USW band using the latest technological advances and highspeed microprocessor technology in RES equipment, the minimum response time $\Delta t_{r \text { min }}$ can be tens of microseconds and less [6, 7]. In these conditions, an important parameter of the FHSS radio-electronic device (in terms of interference immunity) is the actual operating time at one frequency $\Delta t_{o p}$. This parameter, which determines the frequency adjustment rate $v_{\text {adj }}$, characterizes the ability of the FHSS radio device to «escape» from the effects of retransmitted interference.

The factor of signal overlapping by transmitted interference depends not only on the response time $\Delta t_{r}$ but also on the relative location (topology) of the transmitter and the RCD receiver, as well as the station of retransmitted interference on the ground, which determines the delay time of interference $\Delta t_{d}$.

Retransmitted interference is ineffective only on the condition that the radio frequency should be less than the total response time $\Delta t_{o p}$. The delay time $\Delta t_{d}$ of the interference is caused by the location (topology) of the transmitter and the RCD receiver and the station of retransmitted interference on the ground and the final rate of radio wave propagation. In this case, the coefficient $\rho=0$, and the average probability of error per bit is determined only by the receiver's own noise.
Under the action of retransmitted interference, the overlapping factor $\gamma$ is checked. Depending on the $\gamma$ value, there is a change in the operating frequency adjustment rate (step 11, 15). In the absence of retransmitted interference, the FHSS operation mode is chosen for the control of partial band noise interference and noise interference, namely, the coefficient $K_{c h}$ varies depending on the signal/noise ratio according to the step-by-step law with the predefined gradation (stage 12, 16, 17).

4. Correction code selection. By type of noise-proof (corrective) codes, all SCC can be divided into two major classes: on the basis of block codes and on the basis of continuous codes. In addition, a separate class is the SCC based on concatenated codes that use block and continuous codes. Recently, much attention has been paid to the parallel cascading, convolutional codes known as turbo codes [24, 25].

The introduction of turbo codes opened a new direction in solving the problem of creating effective codes and decoding them with little difficulty. These codes provide noise immunity of signal reception by noisy channels close to the theoretically possible (Shannon's boundary).

From [24-26] it is evident that the most effective correcting codes at present are turbo and low-density codes. Each of them has its advantages, disadvantages and, accordingly, its field of application. For example, turbo and low-density codes are capable of operating at a power level of the channel, only a few tenths of a decibel higher than its throughput.

The use of turbo codes during the construction of the SCC allowed for additional energy gain of the signal/noise ratio for channels with fluctuating noise and fading compared to circuits using convolutional codes.

The use of noise immunity encoding increases the width of the signal spectrum, so the code parameters are chosen depending on the bandwidth of the communication channel $\Delta F$.

5. Manipulation code selection. While coordinating the codec of the binary noise immunity code and the modem of multipositional signals, it is necessary to use a manipulation code, in which a greater Hamming distance between the code combinations corresponds to a greater Euclidean distance between the signals corresponding to them.

Methods for coordinating modulation and coding can be divided into two groups: matching with optimal manipulation code and matching on the basis of the ensemble partition into nested sub-ensembles.

The SCCs belonging to the first group are the result of matching the known binary noise immunity codes with the multi-positional signal ensemble by using a specially selected manipulation code. Since errors most often occur due to conversions in neighboring signals, the code combinations that match the neighboring signals should differ in the smallest number of binary characters. This requirement in some cases is satisfied by the Gray code $[25,26]$.

The second group includes a fairly large number of SCC types that differ in the modification of matching methods. The basis of the construction of the SCC of this kind is the partition of the signal ensemble into nested sub-ensembles [25, 26]. The partition is carried out in such a way that the sub-ensembles have the same number of signal points. Euclidean distances $d_{E}$ between the neighboring signals of the sub-ensembles are the same, and the minimum distances $d_{E \text { min }}$ between the sub-ensemble signals are increased with each step of the partition.

Checking compliance with requirements for noise immunity of signal reception. After selecting the manipulation 
code, the requirements for the given probability of erroneous signal reception are checked.

If the requirements $P_{e r}$ are not met, there is a need to change the source data. In the event that the restriction $P_{e r} \leq P_{e r ~ p r o b}$ is met for several SCCs, the SCC with the maximum $\beta_{E}$ values is selected.

\subsection{Evaluating the effectiveness of the proposed} methodology

In order to evaluate the effectiveness of the proposed methodology, simulation of FHSS RCD was carried out at different degrees of deliberate interference affecting the communication channel. The simulation was conducted in the MathCad [26] software environment using the mathematical relationships obtained above.

Fig. 3 shows the graph of the bit error probability against the signal/noise ratio (for the case of frequency shift keying noise barrage interference $\rho=1)$.

The simulation was carried out with the following parameters:

- FHSS radio communication devices: frequency range is $30-512 \mathrm{MHz}$; transmitter power is $10 \mathrm{~W}$; bandwidth of the emitted frequency is $12.5 \mathrm{kHz}$, receiver sensitivity is $110 \mathrm{~dB}$; the number of RCDs in the network is 4; the number of frequency channels for adjustment is 10,000 ; the number of adjustments is 333.5 hops/sec;

- electronic countermeasure complex: frequency range is $30-2,000 \mathrm{MHz}$; transmitter power is $2,000 \mathrm{~W}$; maximum frequency band that can be suppressed is $80 \mathrm{MHz}$; the number of FHSS radio lines, which can be suppressed simultaneously is 4 , type of interference is frequency shift keying noise barrage interference; strategy of the WF complex is dynamic.

To verify the effectiveness of the proposed method, let us suppose that the time of operation of FHSS RCD at the same frequency is the same with the WF complex.

Briefly, we will describe the graphical relationships that are obtained in Fig. 4. As can be seen from Fig. 4, the best approach to the probability of bit error from the signal/noise ratio has been proposed by the authors of the technique. The indicated graphic dependencies were obtained by averaging the signal/noise ratio on the frequency subchannels. This advantage is due to the prediction of the strategy of the WF complex taking into account the influence of transmitters on each other and the choice of optimal SCCs for a separate frequency subchannel. Compared with the method [1], the proposed technique has an average gain of $14.17 \mathrm{~dB}$.

Fig. 5 shows the graph of the bit error probability against the signal/noise ratio (for partial band noise interference with frequency shift keying $\rho=0.5$ ).

Practical verification of the effectiveness of the developed methodology was conducted, by developing the Python 3.6 software (Jupyter-notebook). The software was installed on a PC with 4 programmable LimeSDR transceivers connected to the GNU Radio software and connected to the RIGOL DG5252 noise generator that simulated the operation of the WF complex.

Fig. 6 shows the work of 4 transceivers according to the methodology and RIGOL DG5252 noise generator. As can be seen from Fig. 5, the WF devices is not able to carry out guaranteed suppression of FHSS radio communication devices using this method. For greater visibility, Fig. 6 shows the enlarged image of the work of the indicated method. From Fig. 7 it is possible to conclude that the WF devices of narrowband interference affected about $10 \%$ of the hopset, which can be effectively corrected by correcting codes.

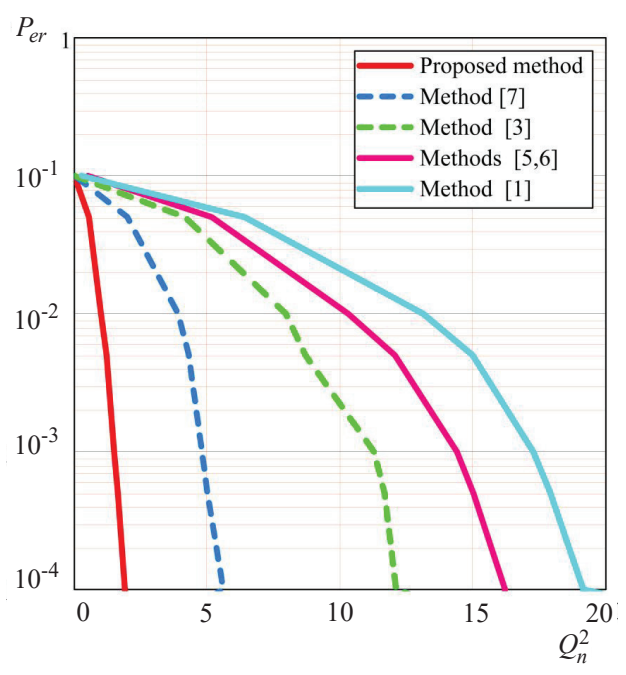

Fig. 4. Dependence of the bit error probability on the signal/noise ratio for different methods under the influence of fluctuation noise and noise barrage interference $(\rho=1)$

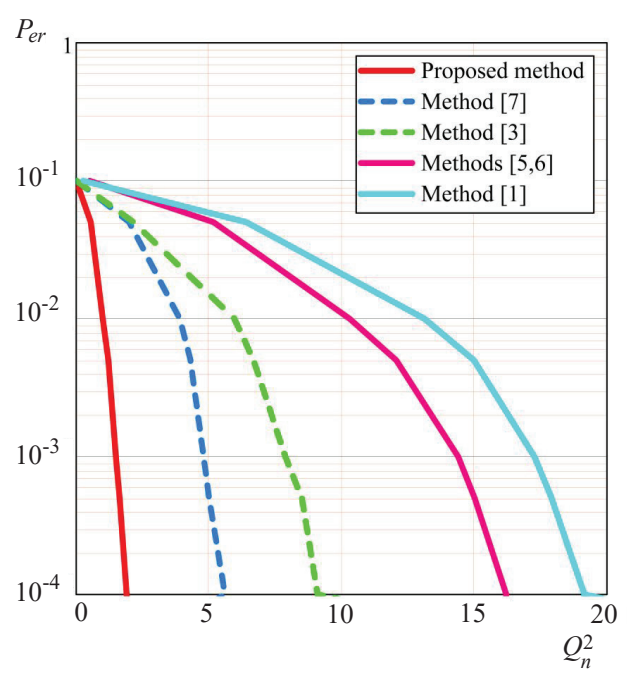

Fig. 5. Graph of dependence of bit error probability on the signal/noise ratio (for the partial band noise interference with frequency shift keying $\rho=0.5$ )

Fig. 8 shows the simulation of the RCD and WF device under the influence of noise interference with frequency shift keying.

Estimating the complexity of the developed methodology showed that for the given output data and while using the ADSP-21261 processor, the formation of a signal with optimal parameter values can be realized in real time. This takes into account the delay required to transmit information about these values through the service feedback channel.

The research of the developed algorithm of adaptive RCD according to the developed method showed that the adaptive scheme of the radio communication devices provides on average 16-27\% higher energy efficiency (utilization rate of signal power) than nonadaptive. 


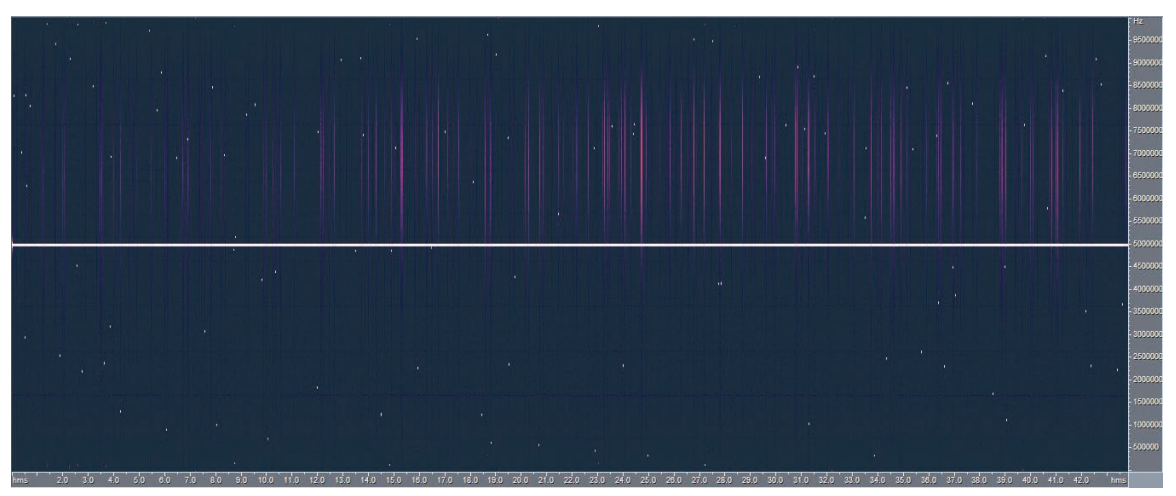

Fig. 6. Operation of 4 transceivers according to the specified methodology and RIGOL DG5252 noise generator
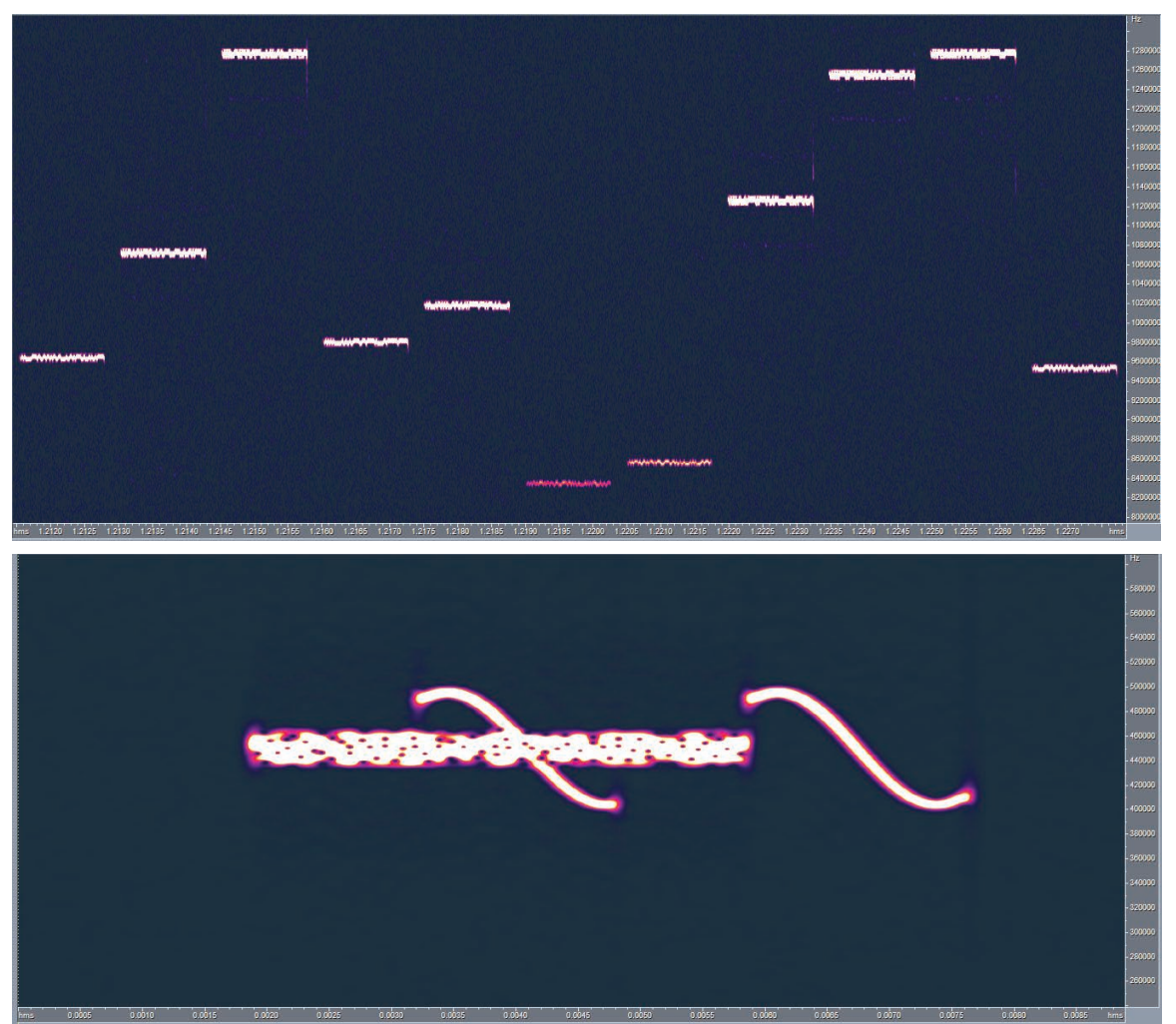

Fig. 7. Operation of 4 transceivers according to the specified methodology and RIGOL DG5252 noise generator

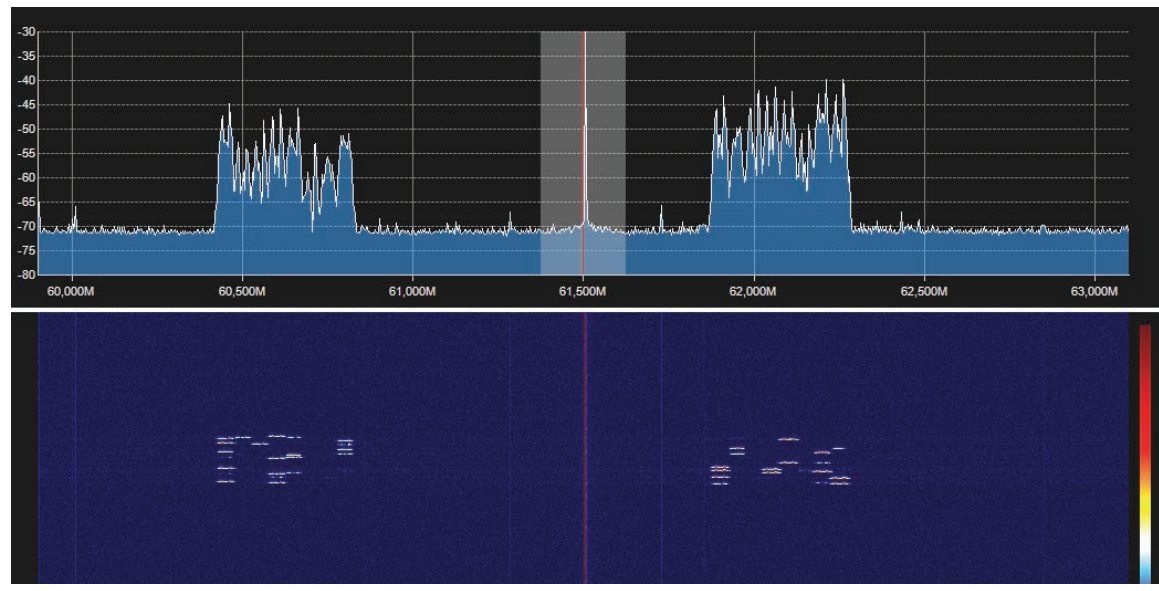

Fig 8. Simulation of the RCD and WF device under the influence of noise interference with frequency shift keying
5. Discussion of the results

of development of a technique for increasing the interference immunity

It has been found that this technique has a higher computational complexity at the level of $10 \%$ compared with others, however, it can increase the interference immunity to $30 \%$ (Fig. 9), which is acceptable. This is due to the fact that this methodology contains additional procedures for calculating the influence of other transceivers of FHSS RCD on each other, as well as the procedure for changing the initial polynomial, which defines the law of adjustment of the operating frequency.

The main advantages of the proposed methodology are:

- an increase of efficiency of radio frequency resource use;

- the possibility of the RCD work in the conditions of the frequency range deficiency;

- a wide scope of use (civil and special use of radio communication systems);

- the possibility of adaptation to the signaling situation in the channel;

- consideration of the impact of the main types of intentional interference;

- the ability to work at low sig$\mathrm{nal} /$ interference ratios in the channel;

- the possibility of frequency-territorial planning of the use of radio communication facilities;

- the possibility of electronic environment forecasting;

- change of the law of FHSS in the network;

- the possibility of synthesizing the optimal structure of the radio communication device.

The proposed method allows increasing the interference immunity of FHSS radio communication devices taking into account a considerable amount of destabilizing factors. This allows solving the contradiction between science and practice, which was formulated in stage 2 of this research, by developing a procedure for the allocation of operating frequencies and an improved assessment of the effect of destabilizing factors.

The disadvantages of the proposed method include:

- increased computing complexity at the level of $10 \%$, which is associated with an increase in the number of procedures; 
- while changing the law of adjustment of the operating frequency, for a certain time there will be a lack of communication;

- this technique is not appropriate for the use in radio communication systems with a small number of correspondents in the network and in radio directions.

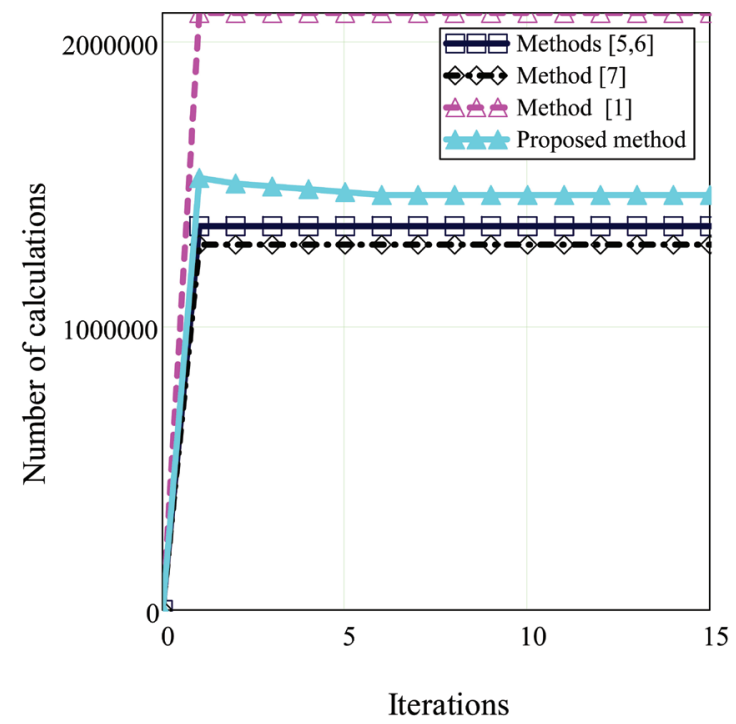

Fig. 9. Analysis of the computational complexity of the proposed methodology

The proposed methodology is advisable to use in developing software for modules (blocks) for evaluating promising radio communication equipment based on the open architecture interfaces of version SCA 2.2, which will allow:

- using efficient signal-code designs to ensure interference immunity of channels;

- ensuring the efficient use of the radio frequency resource of programmable radio communication equipment;

- increasing the speed of communication channels estimation;

- reducing the use of computing resources of radio communication devices with programmable architecture.

It is advisable to use this methodology in radio stations with programmable architecture, which operates in the conditions of active electronic countermeasure.

The mentioned technique will allow:

- identifying the structure of the interference, its type and law of setting;

- evaluating the status of the channel;

- using efficient signal-code designs to ensure interference immunity of channels;

- ensuring the efficient use of the radio frequency resource of programmable radio communication equipment;
- ensuring the efficient use of radio communication devices in the network;

- developing measures aimed at increasing interference immunity.

The proposed methodology should be used in the development of software for modules (blocks) for evaluating promising devices of radio communication, based on the open architecture interfaces of version SCA 2.2.

This study is the further development of research aimed at developing the methodological principles of operational management of radio resources of radio communication systems.

\section{Conclusions}

The method of increasing the interference immunity of frequency-hopping spread spectrum radio communication devices is proposed. The differences of the proposed methodology are:

- the choice of operating frequencies for FHSS RCD is made taking into account the strategy of the electronic countermeasure complexes and taking into account the influence of the RCD transmitters on each other;

- the frequency distribution among the FHSS radio communication equipment is based on the number of RCDs operating on the network;

- Frequency planning is divided into two stages: preliminary and detailed. At the preliminary stage, the choice and analysis of electromagnetic compatibility of FHSS RCD on the frequency-territorial basis is carried out. At the stage of detailed analysis, calculations of electromagnetic compatibility are carried out taking into account the possible influence of the side and intermodulation radiation, as well as the possibility of blocking the receivers when the group of RED is located in a confined space;

- the control of the FHSS parameters is carried out by changing the speed of adjustment of the operating frequency and, if necessary, changing the law of adjustment of the operating frequency;

- taking into account the influence of the most common types of deliberate interference;

- using $M$ positional signal-code structures.

The evaluation of the effectiveness of the proposed method showed an increase in the interference immunity of FHSS radio communication devices up to $30 \%$ while using it.

The proposed method can be implemented in radio communication devices with programmable architecture. To do this, it is necessary to adapt the signal processor through additional software for a specific radio communication device. It is expedient to develop the above-mentioned software on the SCA 2.2 platform.

\section{References}

1. Ivanov M. S., Popov S. A. Pomekhozashchishchennost' shirokopolosnyh sistem radiosvyazi s rasshireniem spektra metodom psevdosluchaynoy perestroyki rabochey chastoty // XI Vserossiyskaya nauchno-prakticheskaya konferenciya «Aktual'nye voprosy razrabotki i vnedreniya informacionnyh tekhnologiy dvoynogo primeneniya». Yaroslavl', 2011. P. 322-329.

2. Chudnov A. M. Ob adaptivnyh algoritmah psevdosluchaynogo pereklyucheniya rabochih chastot radioliniy v usloviyah sluchaynyh i prednamerennyh pomekh // Zhurnal radioelektroniki. 2015. Issue 4.

3. Nahorniuk O. A. Method of automatic time parameters estimation of radio signals with frequency-hopping spread spectrum against the background of narrow-band interferences // Zbirnyk naukovykh prats ZhVI. 2018. Issue 15. P. 53-64. 
4. A Method for Dynamically Selecting the Best Frequency Hopping Technique in Industrial Wireless Sensor Network Applications / Fernández de Gorostiza E., Berzosa J., Mabe J., Cortiñas R. // Sensors. 2018. Vol. 18, Issue 2. P. 657. doi: https://doi.org/10.3390/ s18020657

5. Krivenko A. Method of forming signal in radio facilities with fhss in conditions of influence of intentional noise inferences // Systemy ozbroiennia i viiskova tekhnika. 2017. Issue 1. P. 132-135.

6. Gurski T. G., Krivenko A. V. Method the forming signal of radio communication with FHSS mode voice under influence of noise response // Systemy upravlinnia, navihatsiyi ta zviazku. 2017. Issue 2. P. 179-184.

7. Shishatskiy A., Kuvshinov A., Petrunchak S. Method of selection rational values of parameters of multi-antenna systems of military radio communications with pseudo-random reconstruction of operating frequency // Systemy ozbroiennia i viyskova tekhnika. 2017. Issue 2. P. 151-155.

8. Ershov R. A., Morozov O. A., Fidel'man V. R. Ocenka vzaimnoy vremennoy zaderzhki signalov s psevdosluchaynoy skachkoobraznoy perestroykoy chastoty // Izvestiya vysshih uchebnyh zavedeniy. Radiofizika. 2015. Vol. 58, Issue 2. P. 157-166.

9. Lei Z., Yang P., Zheng L. Detection and Frequency Estimation of Frequency Hopping Spread Spectrum Signals Based on Channelized Modulated Wideband Converters // Electronics. 2018. Vol. 7, Issue 9. P. 170. doi: https://doi.org/10.3390/electronics7090170

10. Kanaa A., Sha'ameri A. Z. A robust parameter estimation of FHSS signals using time-frequency analysis in a non-cooperative environment // Physical Communication. 2018. Vol. 26. P. 9-20. doi: https://doi.org/10.1016/j.phycom.2017.10.013

11. Characterization of anti-jamming effect on the Joint Tactical Information Distribution System (JTIDS) operating in complicated electromagnetic environment / Jin J., Xie H., Hu J., Yin W.-Y. // 2014 International Symposium on Electromagnetic Compatibility. 2014. doi: https://doi.org/10.1109/emceurope.2014.6931048

12. Compressive detection of frequency-hopping spread spectrum signals / Liu F., Marcellin M. W., Goodman N. A., Bilgin A. // Compressive Sensing II. 2013. doi: https://doi.org/10.1117/12.2015969

13. Home of the Coded Modulation Library. URL: http://www.iterativesolutions.com/

14. Recommendation ITU-R P.341-5. The concept of transmission loss for radio links. URL: https://www.itu.int/dms_pubrec/itu-r/ rec/p/R-REC-P.341-5-199910-S!!PDF-E.pdf

15. Kovalenko I. Evaluation method of electromagnetic compatibility of mobile telecommunication means for special purpose // Zbirnyk naukovykh prats. Viiskovoho instytutu telekomunikatsiy ta informatyzatsiyi. 2015. Issue 1. P. 35-41.

16. ICS Telecom. URL: http://lissoft.com.ua/po-atdi/ics-telecom/

17. Method of integral estimation of channel state in the multiantenna radio communication systems / Kalantaievska S., Pievtsov H., Kuvshynov O., Shyshatskyi A., Yarosh S., Gatsenko S. et. al. // Eastern-European Journal of Enterprise Technologies. 2018. Vol. 5, Issue 9 (95). P. 60-76. doi: https://doi.org/10.15587/1729-4061.2018.144085

18. Bandwidth variable transceivers with artificial neural network-aided provisioning and capacity improvement capabilities in meshed optical networks with cascaded ROADM filtering / Zhou X., Zhuge Q., Qiu M., Xiang M., Zhang F., Wu B. et. al. // Optics Communications. 2018. Vol. 409. P. 23-33. doi: https://doi.org/10.1016/j.optcom.2017.09.021

19. Neumann D., Wiese T., Utschick W. Learning the MMSE Channel Estimator // IEEE Transactions on Signal Processing. 2018. P. 1-1. doi: https://doi.org/10.1109/tsp.2018.2799164

20. Nayal N., Sharma K. Channel Estimation in Long Term Evolution // International Journal of Computer Science and Technology. 2015. Vol. 6, Issue 3. P. 230-234.

21. Ivanov M. S., Fedoseev V. E. Technique and results of the analysis of the potential noise stability of reception of the digital signal against the manipulated structural hindrance // Vestnik Voronezhskogo tekhnicheskogo universiteta. 2010. Vol. 6, Issue 11. P. $108-112$.

22. Makarenko S. I. Podavlenie paketnyh radiosetey so sluchaynym mnozhestvennym dostupom za schet destabilizacii ih sostoyaniya // Zhurnal radioelektroniki. 2011. Issue 9. URL: http://jre.cplire.ru/jre/sep11/4/text.pdf

23. Zaitsev S. V. Metod pidvyshchennia dostovirnosti peredachi informatsiyi v rozpodilenykh prohramovanykh bezprovidnykh systemakh z turbokhodamy // Matematychni mashyny i systemy. 2013. Issue 3. P. 142-155.

24. Informatsiyni tekhnolohiyi pobudovy system radiozviazku zi skladnymy syhnalno-kodovymy konstruktsiyamy: monohrafiya / Zaitsev S. V., Kazymyr V. V., Kuvshynov O. V. et. al. Chernihiv: Chernihivskyi derzhavnyi tekhnolohichnyi universytet, 2012. 444 p.

25. Osnovy teoriyi elektrozviazku: pidr. / Ilchenko M. Yu., Korneiko O. V., Kuvshynov O. V., Lezhniuk O. P., Liventsev S. P., Sakovych L. M., Uryvskyi L. M. Kyiv: NTUU «KPI», 2009. 556 p.

26. Shushkevich G. Ch., Shushkevich S. V. Vvedenie v MathCAD 2000: ucheb. pos. Grodno, 2001. 138 p. 